\title{
Saúde Psíquica dos Profissionais de Saúde em Hospitais Públicos
}

\author{
The psychic wellbeing of health personnel working in public \\ hospitals
}

\author{
Luciana C. Barbosa de Oliveira ${ }^{1}$ y Eulália M. Chaves-Maia ${ }^{2}$
} 1 Programa de Pós-graduação em Ciências da Saúde/Hospital de Pediatria Professor Heriberto
Bezerra - Universidade Federal do Rio Grande do Norte. Brasil. lucianacarla.psi@hotmail.com
2 Programa de Pós-graduação em Ciências da Saúde/Departamento de Psicologia - Universidade
Federal do Rio Grande do Norte. Brasil. emcmaia@ufrnet.br.

Recebido 29 Novembre 2007/Enviado para Modificação 19 Maio 2008/Aprovado em 10 Junho 2008

\section{RESUMO}

Objetivo Analisar o nível de saúde psíquica dos profissionais de saúde nas enfermarias dos hospitais públicos do Estado do Rio Grande do Norte, Brasil.

Materiais e Métodos Foi realizado um estudo descritivo transversal com 95 profissionais de saúde (amostra estratificada proporcional). Utilizou-se um questionário constando características sócio-demográficas e de trabalho, assim como o Questionário de Saúde Geral de Goldberg (QSG).

Resultados Os empregados são do sexo feminino, casados, de idade acima de 45 anos. Contam com uma carga horária de trabalho de 40 horas semanais, além de apresentar múltiplos vínculos de trabalho. A saúde psíquica global dos sujeitos encontrase em um nível bom, no entanto, também há indivíduos em número significativo de processo de agravamento, quanto ao stress (37,9\%) e distúrbios psicossomáticos $(34,7 \%)$ isoladamente.

Conclusões Há um indicativo de alerta quanto à necessidade de ações preventivas, de modo a evitar maiores agravamentos com possíveis danos às condições de saúde psíquica destes trabalhadores.

Palavras Chaves: Saúde do Trabalhador, hospitais, ocupações em saúde, saúde mental (fonte: DeCS, BIREME).

\section{ABSTRACT}

Objectives Analysing the level of psychic health in health workers from public hospitals in the State of Rio Grande do Norte, Brazil.

Materials and Methods This was a descriptive, traverse cohort (stratified proportional) study of a sample of 95 health workers; a socio-demographic and work-based questionnaire and the Goldberg's General Health Questionnaire (GGHQ) were used. Results $82 \%$ of the subjects were female, $60 \%$ were married, $81 \%$ catholic, $48 \%$ were aged more than $45,73 \%$ had a workload of 40 hours per week and $63 \%$ worked at other places. $64 \%$ presented a good level of overall psychic health, the psychic health of $31 \%$ of them was becoming affected and $5 \%$ were in extreme danger. 
Conclusions The need for preventative action should be stressed, even with psychically healthy professionals, being, to avoid future damage or more serious worsening of these workers' psychic health.

Keys Words: Health, professional, hospital, mental health (source: MeSH, NLM).

\section{RESUMEN}

\section{La salud psíquica en los profesionales de la salud en hospitales públicos}

Objetivo Analizar el nivel de la salud psíquica de los profesionales de salud en las enfermerías de los hospitales del Estado de Rio Grande do Norte, Brasil.

Material y Métodos Fue realizado un estudio descriptivo transversal con 95 profesionales de salud (muestra estratificada proporcional). Se utilizó un cuestionario constando las características socio-demográficas y laborales, así como el Cuestionario de Salud General de Goldberg (QSG).

Resultados Los empleados son del sexo femenino, casadas, con edad superior a 45 años. Cuentan con una carga horaria laboral de 40 horas semanales, además de presentar múltiples vínculos de trabajo. La salud psíquica global de los sujetos se encuentra en un nivel bueno, pero también hay individuos en número significativo de procesos de agravamiento, en relación al estrés $(37,9 \%)$ y a los disturbios psicosomáticos (34,7\%) independientemente.

Conclusiones Hay un indicativo de alerta sobre la necesidad de acciones preventivas, de modo a evitar mayores complicaciones con posibles daños a las condiciones de salud psíquica de estos trabajadores.

Palabras Clave: Ocupaciones, salud, hospitales, salud mental (fuente: DeCS, BIREME).

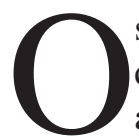

século XXI iniciou carregado de novas perspectivas à melhoria da qualidade de vida e saúde dos indivíduos. No âmbito da saúde os avanços ao que cerne a tecnologia científica são evidentes, este sempre divulgado na mídia. Novos saberes e especialidades também foram acrescidos. Ampliouse a quantidade de hospitais, unidades básicas, postos de saúdes, clínicas, envolvendo uma readequação de espaços, atuações e ações principalmente nos modelos institucionais de atenção à saúde (1).

A instituição hospitalar geralmente é associada a um ambiente complexo, voltado ao atendimento secundário e terciário de pessoas acometidas de doenças que necessitam de internação, desde a mais "simples" a mais complexa. Vinculase este local comumente às rotinas no contexto de enfermarias, cujo período diurno é intenso, marcado pela enorme quantidade de profissionais de saúde exercendo suas funções. São visitas aos pacientes nos leitos, avaliações, prescrições, urgências, exames, entre outros (2). 
Segundo Peduzzi (3) entende-se por profissional de saúde assistencial aquele sujeito no qual desempenha suas atribuições em unidades hospitalares como por exemplo o setor de enfermaria. Neste grupo são várias as categorias inseridas: médicos, enfermeiros, psicólogos, nutricionistas, fisioterapeutas, farmacêuticos, entre outros. Cada um representando a sua respectiva ciência, buscando compreender os "mistérios" de doenças e agravos que acometem a vida humana. No entanto, tais caminhos em busca da saúde, (às vezes) são tortuosos, tanto para o doente quanto para o profissional de saúde (4).

As exigências laborais nos serviços de saúde pública transcorrem desde a produtividade, até a disponibilidade, eficiência e agilidade (5). O número de profissionais é insuficiente à demanda e os salários são pouco atrativos. A sobrecarga no trabalho é uma constante. Conseqüências como baixa produção, insatisfação, aumento do custo operacional e dificuldades de substituições nas escalas já firmadas, são inevitáveis. Tais fatores dificultam não só a qualidade da assistência, mas também trazem prejuízos à qualidade de vida deste trabalhador (6-8).

Segundo análise da Organização Panamericana de Saúde-OPAS, durante o ano de 2001 foram registrados oficialmente no Brasil, em diversos profissionais atuantes no campo da saúde, 25.784 casos de acidentes, doenças e óbitos relacionados ao trabalho (9). Políticas direcionadas à qualidade de vida no trabalho ainda são ineficazes, cujos incentivos à realização de novas pesquisas são escassas. O Ministério da Saúde do Brasil (MS) enfatiza tal necessidade ao perceber o surgimento: "de novas formas de adoecimento mal caracterizadas, como o estresse e a fadiga física e mental e outras manifestações de sofrimento relacionadas ao trabalho. Configura, portanto, situações que exigem mais pesquisas e conhecimentos para que se possa traçar propostas coerentes e efetivas de intervenção" (9).

Falar em saúde no trabalho ainda é algo relativamente novo. No Brasil, foi com o pesquisador Dejour, que os aspectos psicopatológicos no ofício obtiveram uma maior evidência. A obra: A loucura do Trabalho, publicada pela primeira vez em 1987 ainda é considerada um marco no Brasil e na América Latina (10). Trouxe em seu enfoque sobre a psicopatologia do trabalhador (sofrimento, ansiedade, stress, somatizações e doenças). Contudo, o tema sofrimento psíquico relacionado diretamente aos profissionais de saúde ganha uma compreensão mais abrangente com a autora Pitta, ao afirmar: "a noção de sofrimento psíquico designa esta difusa zona entre a saúde e a doença significando um conceito mais preciso no âmbito da psicopatologia do trabalho" (1). Atualmente pesquisas voltadas 
ao campo da saúde do trabalhador vêm crescendo, no entanto ainda são insuficientes. Temas como stress, burnout, ergonomia e saúde psíquica vão aos poucos adquirindo o devido espaço no meio acadêmico e profissional, cujo algumas especialidades como: policiais, docentes, e profissionais de saúde, encontram-se em maior evidência em virtude do perfil laboral (11-15).

Diante dos fatores supracitados relativo aos trabalhadores da saúde, que exercem seu ofício em enfermarias, percebe-se a diversidade de fatores que podem influenciar no nível de stress destes sujeitos. Neste sentido, torna-se pertinente que estas instituições (baseados em estudos) lancem também uma atenção à saúde do profissional de saúde, já que este tem em seu ofício o cuidar do outro (16-18). Esta pesquisa objetivou analisar a saúde psíquica geral e seus fatores específicos junto aos profissionais de saúde atuantes em enfermarias em hospitais do Rio Grande do Norte/Brasil.

\section{MATERIAIS E MÉTODOS}

Se trata de um estudo descritivo qualitativo e quantitativo de corte transversal junto aos profissionais de saúde (médicos, enfermeiros, psicólogos, assistentes sociais e nutricionistas) atuantes nas enfermarias em seis hospitais públicos (03 estaduais e 3 federais), referenciados ao Sistema Único de Saúde (SUS), de pequeno/médio porte, localizados no Estado do Rio Grande do Norte, Brasil; cujo objetivo principal esteve em avaliar o nível de saúde psíquica e suas variáveis.

A seleção da população estudada foi realizada através de uma amostragem aleatória proporcional um "n" de 95 sujeitos válidos, possibilitando assim uma margem de confiança de 95 \%. Ressalta-se aqui a consideração da visão global dos aspectos inerentes aos profissionais na análise dos resultados. Para os critérios de inclusão da amostra foram ponderados: a vinculação funcional concursado no hospital por no mínimo de dois anos; estar em exercício profissional em cargo de nível superior sem desvio de função, e desempenhar suas atribuições junto ao setor de enfermaria e estar enquadrado em escalas de plantão diurno (pela dinâmica configurar-se como mais ativa geralmente). Os sujeitos que não contemplassem todos estes aspectos ou se recusassem a participar do estudo, estariam automaticamente excluídos da amostra. Vale salientar, que para acesso a tais dados referente à amostra, o referido estudo contou com o apoio do Setor de Recursos Humanos de cada instituição.

Este trabalho foi aprovado seguindo os padrões e normas estabelecidas pelo Comitê de Ética em Pesquisa da Universidade Federal do Rio Grande do Norte 
em cumprimento à Resolução No. 196/96 do Conselho Nacional de Saúde/ BRASIL.

Os instrumentos foram aplicados junto à amostra de forma individual, no local de trabalho, durante o intervalo das suas atribuições, entre novembro de 2006 a junho de 2007 utilizando um questionário semi-aberto contemplado por dados sócio-demográficos, informações pessoais e profissionais; e o Questionário de Saúde Geral de Goldberg (QSG) (20). Tal método consiste em uma avaliação psicológica, composta por 60 itens cujo objetivo está em avaliar os sintomas psíquicos através dos seguintes fatores: F1-Tensão ou stress psíquico; F2-Desejo de Morte; F3-Falta de confiança na capacidade de desempenho (auto- eficácia); F4-Distúrbios do sono; F5-Distúrbios psicossomáticos; e, F6-Ausência de saúde psíquica (fator geral). O teste em questão possibilita avaliar a saúde específica dentre cada fator supracitado, assim como (dependendo da necessidade) analisar como único fator geral (F6), demonstrando dimensões gerais da ausência de saúde mental (sofrimento psíquico). O referido instrumento foi validado no ano de 1972 e adaptado à realidade brasileira em 1996 (19). Vale ressaltar que este teste psicológico é devidamente reconhecido e autorizado pelo Conselho Federal de Psicologia do Brasil.

Para análise e tratamento dos dados contou com o manual e crivos de correção (QSG), e o programa estatístico SPSS (Statistical Package for Social Science for Windows, versão 12.0).

\section{RESULTADOS}

\section{Caracterização}

A amostra deste estudo encontrou-se caracterizada por enfermeiros (33,6 \%) médicos (28,4 \%), nutricionistas (16,8 \%), assistentes sociais (15,7 \%), e 5,2 \% de psicólogos. Destes, 82,1 \% são do sexo feminino, casados (58,9\%), católicos (80,0 \%) e com uma faixa etária predominantemente acima de 45 anos (47,3\%). Com relação à instituição vinculada à carga horária disposta ao trabalho local é bem representativa considerando que 73,6 \% trabalham uma média de 40 horas semanais nos hospitais. Também foi questionado, a quantidade de folga/dia que eles dispõem para se dedicarem à vida pessoal encontrando 54,7 \% de sujeitos que contam com dois dias disponíveis e 26,3 \% contam somente com um dia. Muitos profissionais (62,1\%) colocam a necessidade de se trabalhar em outro local, de modo a poder complementar a renda da família. Vale salientar que destes outros vínculos $25,2 \%$ tem 2 ou mais outros empregos. Cabe aqui colocar 
que 49,4 \% da amostra recebem mensalmente entre 4 a 6 salários mínimos brasileiros (atualmente a média do salário mínimo no Brasil é o equivalente a 204,63 dólares).

Avaliação da saúde psíquica

Esta avaliação pode ser analisada tanto por fatores (F) específicos: Tensão ou Stress Psíquico (F-1), Desejo de Morte (F-2), Falta de Confiança na Capacidade de Desempenho/auto - eficácia (F-3), Distúrbios do Sono (F-4), Distúrbios Psicossomáticos (F-5), e finalmente a Saúde Psíquica Geral (F-6).

O perfil sintomático é expresso por fatores de saúde cujos escores se situam com valores de 5 a $100 \%$. Os casos que acusem um acima do percentil 90, referem-se aos sujeitos com a saúde gravemente debilitada, necessitando de tratamentos. Percentuais demonstrados entre 55 próximo a 90 são considerados limítrofes (em processo de agravo, necessitando de atenção). Já os indivíduos que apresentaram percentiis abaixo de 50 demonstram apresentar em saúde psíquica entre boa a ótima.

Tendo em vista a sitomatologia apresentada na Tabela 1 percebe-se em seus fatores específicos de stress psíquico, desconfiança do próprio desempenho, distúrbio do sono e distúrbio psicossomático, a presença de um quantitativo relevante de sujeitos limítrofes.

Tabela 1. Perfil sintomático de saúde psíquica e seus fatores específicos, em profissionais de saúde

\begin{tabular}{|c|c|c|c|c|c|c|c|c|c|c|c|c|}
\hline \multirow{3}{*}{$\begin{array}{l}\text { Fatores } \\
\text { Valor }\end{array}$} & \multicolumn{10}{|c|}{ Questionánio de Saúde Geral de Goldberg (QSG) } & \multirow{2}{*}{\multicolumn{2}{|c|}{$\mathrm{F} 6^{9}$}} \\
\hline & \multicolumn{2}{|c|}{$F 1^{6}$} & \multicolumn{2}{|c|}{$\mathrm{F}^{2}$} & \multicolumn{2}{|c|}{$\mathrm{F}^{\mathrm{a}}$} & \multicolumn{2}{|c|}{$\mathrm{F} 4^{*}$} & \multicolumn{2}{|c|}{$\mathrm{F} 5^{1}$} & & \\
\hline & $\mathbf{n}$ & $\%$ & $\mathbf{n}$ & $\%$ & n & $\%$ & $\mathbf{n}$ & $\%$ & n & $\%$ & n & $\%$ \\
\hline $5-45^{\prime}$ & 46 & 48,4 & 69 & 72,6 & 42 & 44,2 & 41 & 43,1 & 39 & 41,0 & 61 & 64,2 \\
\hline $50^{\prime}$ & 2 & 2,1 & 0 & 0 & 5 & 5,2 & 0 & 0 & 5 & 5,2 & 0 & 0 \\
\hline $55-85$ & 36 & 37,9 & 20 & 21,0 & 35 & 36,8 & 34 & 35,8 & 33 & 34,7 & 29 & 30,5 \\
\hline $90-100^{k}$ & 11 & 11,6 & 6 & 6.3 & 13 & 13,7 & 20 & 21,0 & 18 & 18.9 & 5 & 5.2 \\
\hline Total & 95 & 100 & 95 & 100 & 95 & 100 & 95 & 100 & 95 & 100 & 95 & 100 \\
\hline
\end{tabular}

a. Amostra correspondente a $n=95$ profissionais de saúde. Valor e análise conforme padrões recomendados e estabelecidos pelo Questionário de Saúde Geral de Goldberg (QSG); ${ }^{\text {b. }} \mathrm{F}$ 1-Tensão ou stress psíquico; ${ }^{\text {c }}$.

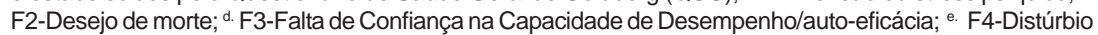

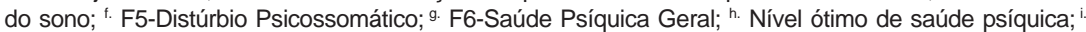

Nível bom de saúde; '. Limítrofe, em processo de agravo, e; ${ }^{k}$. Sintoma de sofrimento.

O fator stress (F-1) especificamente, pode afetar não somente a saúde mas também ocasionar reflexos na qualidade laboral gerando inseguranças nos desempenhos dos trabalhadores, conforme demonstrado no Fator 3. No que se refere aos fatores de distúrbios do sono (F-4) e psicossomático (F-5), também 
foi encontrado um número significativo de indivíduos cujos sintomas estão em estado muito grave.

Ao se avaliar o nível de saúde psíquico geral da amostra (F-6), foi constatado que $64,2 \%$ apresentam um bom estado, 30,5 \% estão na categoria de agravamento, recomendando-se atenção e 5,2 \% apresentam sofrimento psíquico em seu estado mais avançado. Apesar desta benesse, nesta avaliação geral de saúde, também cabe a inserção de ações de cunho preventivo enfocando os fatores específicos significativos, buscando o não agravamento destes sujeitos, assim como o devido encaminhamento assistencial àqueles que estão em seu processo mais difícil, com doenças já instaladas.

\section{DISCUSSÃO}

Considerando o perfil dos participantes, podese afirmar que é perceptível a presença prevalecente do sexo feminino, não diferenciando de alguns estudos com cujo tal fator podendo estar influenciado devido a quatro das cinco profissões (Psicologia, Serviço Social, Nutrição e Enfermagem) terem em sua natureza um caráter predominantemente de gênero feminino (1,5-7).

A sobrecarga de trabalho é evidente, considerando que grande parte apresentou uma carga horária semanal extensa na instituição, corroborando assim, com dados encontrados na literatura $(1,3,5)$. Outro fator aqui relevante mostra-se com a necessidade dos profissionais em trabalhar em outros locais para complementar a renda, não diferenciando de outros estudos $(7,12)$. Neste contexto, os referidos sujeitos também exercem atividades, concomitantemente em outras instituições, (graças ao turno flexível) característico de sua atividade profissional em meio a plantões. Sabe-se que o excesso de trabalho dá margem à insatisfação, desestímulo e cansaço, ainda mais que os horários alternados de plantões não possibilitam ao profissional um espaço para descanso, dada à imprevisibilidade da demanda e do fluxo constante de solicitações por parte dos pacientes (14-16).

Ser profissional de saúde não é uma tarefa fácil. Além da pressão diária trazida pelas atribuições, existem outros aspectos inerentes que influenciam na sua saúde emocional. Dentre os sujeitos do estudo, a saúde psíquica deles de um modo geral encontra-se em um nível positivo, no entanto 31 \% apresentam um nível de agravamento em seu estado.

O lidar com a dicotomia vida-morte junto ao paciente, faz com que sentimentos e emoções como culpa, raiva, angústia, compaixão, impotência e até mesmo a 
onipotência estejam sempre presentes. Não é fácil lidar com o sofrimento humano. As somatizações ocorrem com freqüência, como foi possível perceber que 34,7 \% enquadram-se nos percentis de 55 a 85 \%, requisitando uma atenção a sua saúde. Vale salientar que neste fator específico 18,9 \% apresentaram gravidade extrema, sendo indicativo a tratamento. Nesta fase, sintomas como exaustão, fadiga, tensão muscular, cefaléias, distúrbios do sono que, dependendo do caso, podem se agravar através de patologias como câncer, hipertensão, distúrbio gastrointestinais, transtornos mentais, entre outros (17-19).

Diante dos aspectos supracitados, vale ainda salientar que é importante que os gestores tenham a consciência do reflexo positivo que estes aspectos supracitados incidem sobre a qualidade de vida no trabalho. Sabe-se ainda que a conduta que um profissional de saúde pode ter sobre o paciente requer não só uma de qualidade, mas também exige condições humanizadas no hospital de uma forma mais ampla $(5,7,9,14)$.

Com os resultados expostos foi possível observar que a realidade da saúde é um tanto precária de modo que, inseridos em um hospital, é possível encontrar não só sujeitos doentes em tratamento, mas também alguns dos próprios profissionais (cuidadores) com sua saúde debilitada, (necessitando de cuidados e atenção) em pleno exercício de suas atribuições: o cuidar do outro. Diante da vulnerabilidade em que o indivíduo apresenta, torna-se viável se propor um melhor direcionamento de tratamentos ou ações preventivas a estes sujeitos, cujo enfoque esteja direcionado à redução do stress, qualidade de vida e ergonomia no trabalho.

Devido ao estudo sofrer limitações quanto à especificidade do ambiente de enfermaria, é muito importante acrescentar em novas pesquisas englobando outros locais como UTI, pronto-socorro e urgência, assim como a inserção de outros profissionais da área. Esta pesquisa de carater inédito nos hospitais do Estado do Rio Grande do Norte trouxe subsídios para realização de futuras pesquisas voltadas tanto à saúde destes indivíduos, visto que estudos neste campo ainda são escassos e precisam ser enfatizados.

Percebe-se que o sofrimento psíquico no trabalho pode vir através da apresentação de pequenos sinais e sintomas (sofrimento, ansiedade, irritação, agressividade, stress, somatizações) até chegar à própria doença. A saúde mental de quem lida com conteúdos ansiogênicos e estressantes no dia a dia faz jus a uma vigilância efetiva e eficaz. Tal necessidade urge, na medida em que, se não oferecer melhores condições de saúde a quem "faz" saúde, este não terá condições de "reproduzir" saúde ao próximo * 
Agradecimentos: Ao apoio e incentivo proporcionado pelos: Grupo de Estudos: Psicologia e Saúde, Programa de Pós-Graduação em Ciências da Saúde e Hospital de Pediatria Professor Heriberto Bezerra - Universidade Federal do Rio Grande do Norte; Priscilla de Araújo, Tabita Moreira, José Hélder de Aquino, Camomila Ferreira e Daniella Faria pela colaboração durante a realização deste estudo.

\section{REFERÊNCIAS}

1. Pitta AMF. Hospital: dor e morte como ofício. $3^{a}$ ed. São Paulo: Hucitec; 1999.

2. Angerami-Camon VA. Psicologia da Saúde - Um Novo Significado para a Prática Clínica. São Paulo: Pioneira; 2000.

3. Peduzzi M. Equipe Multiprofissional de Saúde: conceito e tipologia. Revista Saúde Pública. 2001; 35 (1), 103-109.

4. Martins P. Contra a Desumanização da Medicina: crítica sociológica das práticas médicas modernas. Petrópolis: Vozes; 2003.

5. Palácios M. A saúde mental de quem trabalha em saúde: o caso de um hospital geral do Rio de Janeiro. Cadernos IPUB: organização do trabalho e saúde mental. 2000; 2 (1). 52-66.

6. Romero AB. Personal de Enfermería: condiciones de trabajo de alto riesgo. Salud de los Trabajadores. 1998; 6 (2). 113-119.

7. Organização Mundial de Saúde. Atlas Global da Força de Trabalho em Saúde [Internet]. Disponível em: URL: http://www.who.int/globalatlas/default.asp. Consultado em dezembro de 2006.

8. Costa ES, Martinez MAR. Percepção dos efeitos do trabalho em turnos sobre saúde e a vida social em funcionários da enfermagem em um hospital universitário do Estado de São Paulo. Cadernos de Saúde Pública. 2000; 16 (2), 553-555.

9. Brasil, Ministério da Saúde. Organização Pan-americana de Saúde no Brasil. Doenças relacionadas ao trabalho: manual de procedimentos para os serviços de saúde. Brasília: Ministério da Saúde do Brasil; 2001.

10. Dejours C. A Loucura do Trabalho: estudo sobre a psicopatologia no trabalho. São Paulo: OBOR; 1997.

11. Costa M, Accioly Jr H, Oliveira J, Maia E. Estresse: diagnóstico dos policiais militares em uma cidade brasileira. Rev. Panam. Salud Publica. 2007; 21 (4): 217-22.

12. Lacaz FAC. Qualidade de vida no trabalho e saúde/doença. ABRASCO-Associação Brasileira de Pós- Graduação em Saúde Coletiva. 2000; 5 (1): 151-161.

13. Bermúdez PB, Burillo JMT, Martinez NT, Rentero BD, Viguer Z. Accidentes de Trabajo en un hospital de agudos. Revista Española de Salud Publica. 1998, 72 (2), 127-136.

14. De Marco MA. A face humana da medicina: do modelo biomédico ao modelo biopsicossocial. São Paulo: Casa do Psicólogo; 2003.

15. Restrepo-Ayala NC, Colorado-Vargas GO, Cabrera-Arana GA. Desgaste Emocional en Docentes Oficiales de Medellín, Colombia, 2005. Rev. Salud Pública -Bogotá, Colombia. 2006; 08 (1): 63-73.

16. Borges LO. Os profissionais de saúde e seu trabalho. São Paulo: Casa do Psicólogo; 2005.

17. Organización Mundial de la Salud. La organización del trabajo y el estrés: estrategias sistematicas de solución de problemas para empleadores, personal directivo y representantes síndicales. Série protección de la salud de los trabajadores. Genebra: OMS; 2004.

18. Juárez-García A. Factores psicosociales laborales relacionados con la tensión arterial y síntomas cardiovasculares en personal de enfermería en México. Salud Pública de México. 2007; 49 (2): 109-117.

19. Pasquali L, Gouveia VV, Andriola WB, Miranda FJ, Ramos ALM. Questionário de Saúde Geral de Goldberg (QSG). São Paulo: Casa do Psicólogo; 1996. 